\title{
The Classification of Elbow Extension and Flexion: A feature selection investigation
}

\author{
Mohamad llyas Rizan¹, Muhammad Nur Aiman Shapiee ${ }^{1}$, Muhammad Amirul Abdullah¹, Mohd Azraai Mohd Razman ${ }^{1}$ and Anwar \\ P.P. Abdul Majeed ${ }^{*}$ \\ ${ }^{1}$ Faculty of Manufacturing and Mechatronics Engineering Technology, Universiti Malaysia Pahang, 26600 Pahang, Malaysia.
}

ABSTRACT - Nowadays, the worldwide primary reasons of long-term disability is stroke. When the blood supply to your brain is interupted and reduced, stroke occurs as it depriving brain tissue of nutrients and oxygen. In the modern world, advanced technology are revolutionizing the rehabilitation process. This research uses mechanomyography (MMG) and machine learning models to classify the elbow movement, extension and flexion of the elbow joint. The study will aid in the control of an exoskeleton for stroke patient's rehabilitation process in future studies. Five volunteers (21 to 23 years old) were recruited in Universiti Malaysia Pahang (UMP) to execute the right elbow movement of extension and flexion. The movements are repeated five times each for two active muscles for the extension and flexion motion, namely triceps and biceps. From the time domain based MMG signals, twenty-four features were extracted from the MMG before being classified by the machine learning model, namely $k$-Nearest Neighbors $(k-$ $\mathrm{NN}$ ). The $k$-NN has achieved the classification accuracy (CA) with $88.6 \%$ as the significant features are identified through the information gain approach. It may well be stated that the suggested process was able to classify the elbow movement well.

\section{ARTICLE HISTORY}

Received: $7^{\text {th }}$ Nov 2020

Revised: 2nd Dec 2020

Accepted: $17^{\text {th }}$ Dec 2020

\section{KEYWORDS}

Mechanomyography

Elbow movement

Machine Learning

Classifier models

Classififcation

\section{INTRODUCTION}

Stroke is a primary factor of death and incapacity international, and the monetary charges of post-stroke care and treatment are significant. The Global Burden made a systematic approach of Diseases, Injuries, and Risk Factors Study (GBD), which practices using a technique of evaluating wellbeing by sickness, sex, year, age, and area. It provides data to policymakers and health systems in excess of 300 reasons for illness and damage, including strokes [1]. Consistently, 15 million individuals overall endure a stroke every year. Above $85 \%$ of the patient survived, but just $10 \%$ that was recovered totally. Others would like a pact with mobility impairments in the upper and lower limb, cognitive disabilities or numerous different kinds of post-stroke conditions. Robotic systems are progressively utilised in rehabilitation for stroke patients. The results of controlled trials involving human subjects affirm the effectiveness of robot-enhanced techniques and turn out to be gradually better over standard manual treatment in some cases. Exoskeletons have been broadly examined because of their capacity to give paretic limbs more control, just as the complexities engaged with their design and control [2].

Two main methods are used to acquire the muscle signals: Electromyography (EMG) and Mechanomyogram (MMG). MMG is the mechanical sign noticeable from the outside of a muscle when the muscle is contracted. Toward the start of muscle constriction, net changes in the muscle shape cause an enormous crest in the MMG. Subsequent vibrations are because of motions of the muscle fibres at the reverberation recurrence of the muscle. EMG is an electrodiagnostic medicine procedure for recording and assessing the electrical action created by skeletal muscles. Electromyograph is an instrument that creates an electromyogram that has been utilising to performed the EMG. Electromyograph will recognise the electric potential formed by muscle cells when these cells are neurologically or electrically stimulated. The signals could distinguish therapeutic differences from the recruitment order, norm, initiation level, and analysis of animals' human development or biomechanics.

Both MMG and EMG could accomplish comparable motion classification performance, providing classification errors of $9.9 \%$ and $7.4 \%$, individually. In control of the Prosthetic System in Multiple Limb Positions for position classification, MMG accomplished essentially lower classification error of $0.7 \%$ compared to $16.5 \%$ when utilising EMG [3]. The demand for less complex, more efficient, and cost-effective devices is high, so MMG equipment is favoured compared to EMG equipment due to the lessening equipment complexity. The inherent absence of $50 \mathrm{~Hz}$ noise, the lesser effect of attenuation by fatigue and the usage of gel-less sensors [4].

The statistical models that computer systems use to effectively perform a specific task and the study of the algorithm, without utilising explicit instruction, depending on inference and patterns, is known as Machine learning (ML). This is viewed as a division of Artificial Intelligence (AI). ML's main objective is to leverage statistical analysis to predict an acceptable output value and build algorithms to get input data. ML can be used in numerous applications, namely publishing, financial sector and banking, healthcare and social media, that permits efficient utilisation of resources and reduction of time cycle [5]. 
The rest of this paper is structured as follows, beginning with the methodology undertaken in this study, experiment setup, and ML models concepts to achieve the desired results. Then followed by reporting the results as well as the associated discussions and lastly, provide the conclusion of the overall research of this paper.

\section{METHODOLOGY}

\section{Experimental Setup}

Five volunteers were recruited from Universiti Malaysia Pahang (UMP. They were required to perform extension and flexion of the right elbow, with all the movements needing to be repeated five times per movement as in Figure 1. In total, 50 readings need to be taken to develop machine learning models. The MMG device will be placed at the biceps and triceps to take the muscle signals as the time reading was set to $100 \mathrm{~ms}$.

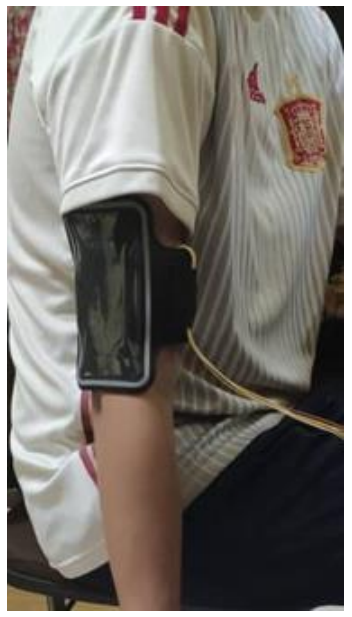

(a)

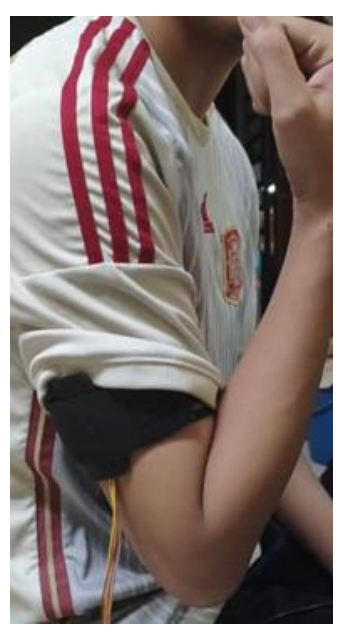

(b)

Figure 1. (a) Extension of Elbow (b) Flexion of Elbow

\section{Features Extraction}

Muscle signals obtained from the device will be calibrated with gravity as a unit reference to make the device function as accurately and error-free as much as possible. Gravitational force or " $G$ " will be the measuring unit which $1 \mathrm{G}$ represents the gravitational pull on the earth's surface. Next, the signals will undergo a feature extraction process to get better precision and accuracy. In this part, features such as mean absolute value (MAV), variance (VAR), standard deviation (STD), and root-mean-square (RMS) were extracted from the filtered signal to be classified accordingly [6]. For each feature, there will be three separations for the $\mathrm{x}, \mathrm{y}$ and $\mathrm{z}$-axis. In total, there will be 24 features which have a total of 50 data.

\section{Machine Learning Technique}

ML is an application of AI that teaches computer systems to execute a particular task without using explicit orders that rely instead on inferences and patterns. We use this technique because it propositions smooth possibility to processing vast volumes of data. ML also could deliver accurate analysis and results by producing efficient and fast algorithms and data-driven models for data processing in real-time.

\section{Classifier}

This research applies the machine learning technique, specifically $k$-Nearest Neighbors ( $k$-NN), to validate all the features via Orange software. Then all the classifiers are evaluated in the test and score widget by displays a table with different performance measures for the classifier such as Classification Accuracy (CA), Area Under Curve (AUC), Recall, precision and F1-score. However, in this study, only the CA and the confusion matrix are evaluated using 10 fold Cross-Validation (CV) on training on testing and training set to obtain the score.

\section{$k$-Nearest Neighbors $(k-N N)$}

The $k$-NN algorithm undertakes that similar things exist in close proximity. It is also a very efficient non-parametric method that can be used to solve both regression and classification difficulties. The $k$-NN method also develops into several variations because of the perfect mathematical theory in the algorithm [7]. The input consists of the $k$ closest training examples in the feature space, and the output will depend on classification or regression. The number of neighbours was set to 3 in $k$-NN for this study. The distance was set as uniform, and metric was set to Euclidean. 


\section{Sampling Methods}

In this study, there were many types of sampling methods used. The first is a fixed proportion of data that splits the entire data $70 \%$ to training data and the remaining $30 \%$ to testing data. Next is to tests on training data and tests on testing data. Test on training data often gives wrong results as it uses all the dataset input to training then testing it. While testing on testing data was to input other data as testing data, the K-Fold CV technique was used to divide the data into folds, $\mathrm{k}$ subsets, as in Figure 2. ML model is trained on all but one $(\mathrm{k}-1)$ of the folds, then the model on the fold that was not used for training was evaluated. This process is repeated $\mathrm{k}$ times, with a different fold reserved for evaluation and excluded from training each cycle. The K-Fold used in this study is 10 Fold.

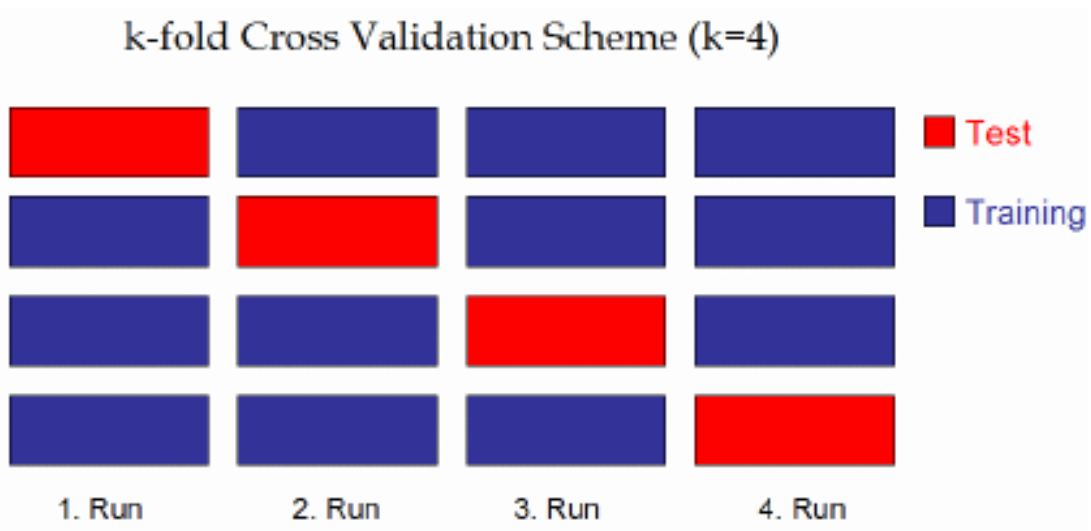

Figure 2. Example 4-fold CV

\section{Confusion Matrix}

Confusion matrix as in Table 1 has often been used to represent the results of a classification model on a set of test data that the true values are known. It provides the number or proportion of instances between the predicted and actual class. Thus it is possible to determine which specific instances have been misclassified.

Table 1. Confusion Matrix Table

\begin{tabular}{ccc}
\hline & Predicted Positive & Predicted Negative \\
\hline Actual Positive & TP & FN \\
Actual Negative & FP & TN \\
\hline
\end{tabular}

The entries in the table above have the following meanings:

- $\quad \mathrm{TP}$ is the number of true positive predictions

- $\quad \mathrm{FP}$ is the number of false positive predictions

- $\mathrm{TN}$ is the number of true negative predictions

- $\mathrm{FN}$ is the number of false negative predictions

Model performance is often computed from a confusion matrix where Classification Accuracy (CA): the proportion of classifier that was correct.

$$
\text { Accuracy }=\frac{(T P+T N)}{(T P+T N+F P+F N)}
$$

\section{EXPERIMENTAL RESULTS}

\section{Data Acquisition}

For data acquisition, Figure 3 below will show the extension movement of the right elbow. The muscle signals obtained from the MMG device also display in the $\mathrm{x}, \mathrm{y}$ and $\mathrm{z}$-axis for two muscles, biceps and triceps, in the elbow movement depicted in Figures 4 and 5, respectively. The measurements were taken in terms of g, i.e., $1 \mathrm{~g}$ is equivalent to $9.81 \mathrm{~m} / \mathrm{s} 2$. 


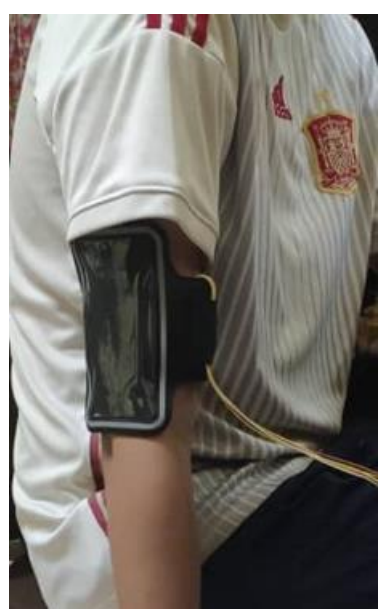

(a)

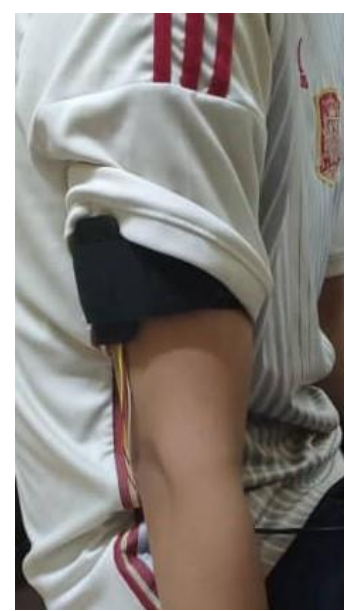

(b)

Figure 3. (a) Extension for Biceps muscle (b) Extension for Triceps muscle

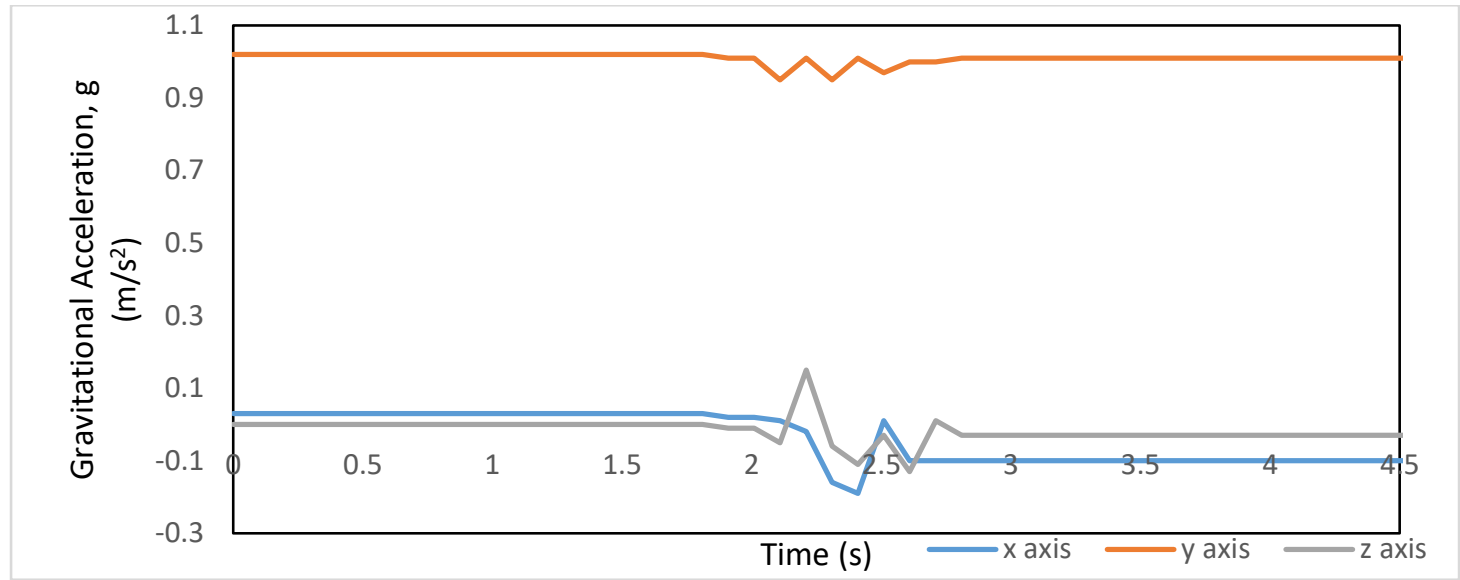

Figure 4. Signal for Extension Biceps muscle

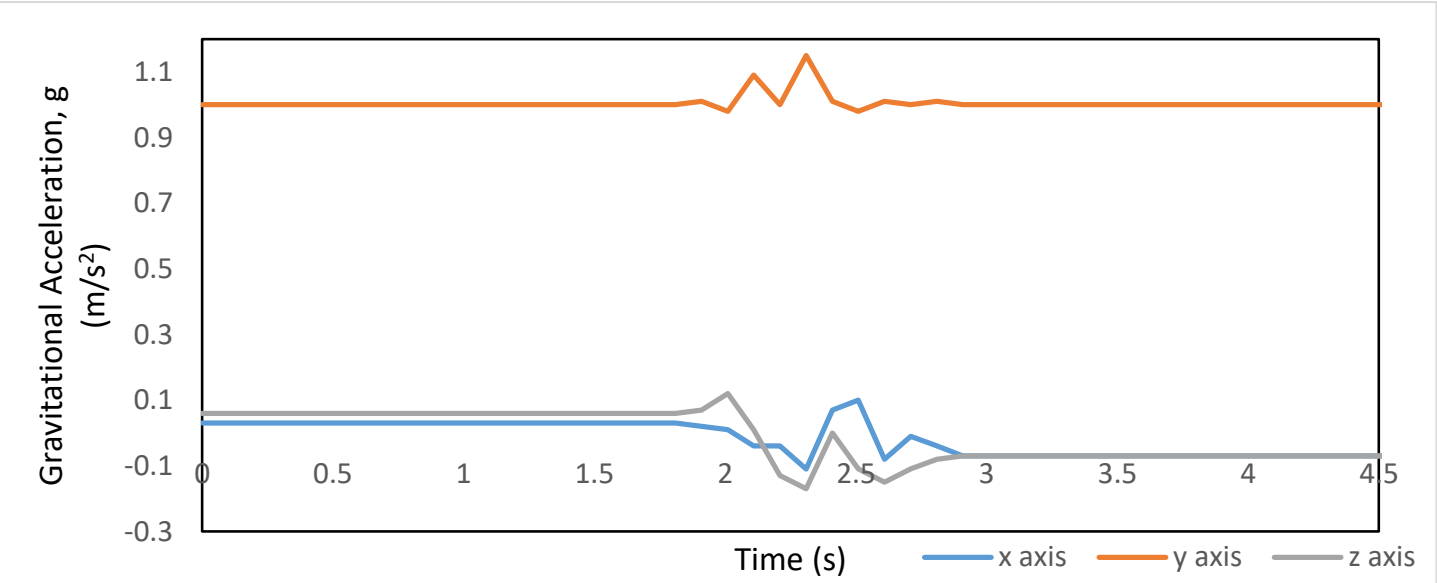

Figure 5. Signal for Extension Triceps muscle

For flexion movements, Figure 6 illustrates the flexion movement of the right elbow. The muscle signals obtained from the MMG device also display in the $\mathrm{x}, \mathrm{y}$ and $\mathrm{z}$-axis for two muscles, biceps and triceps, in the elbow movement depicted in Figures 7 and 8, respectively. 

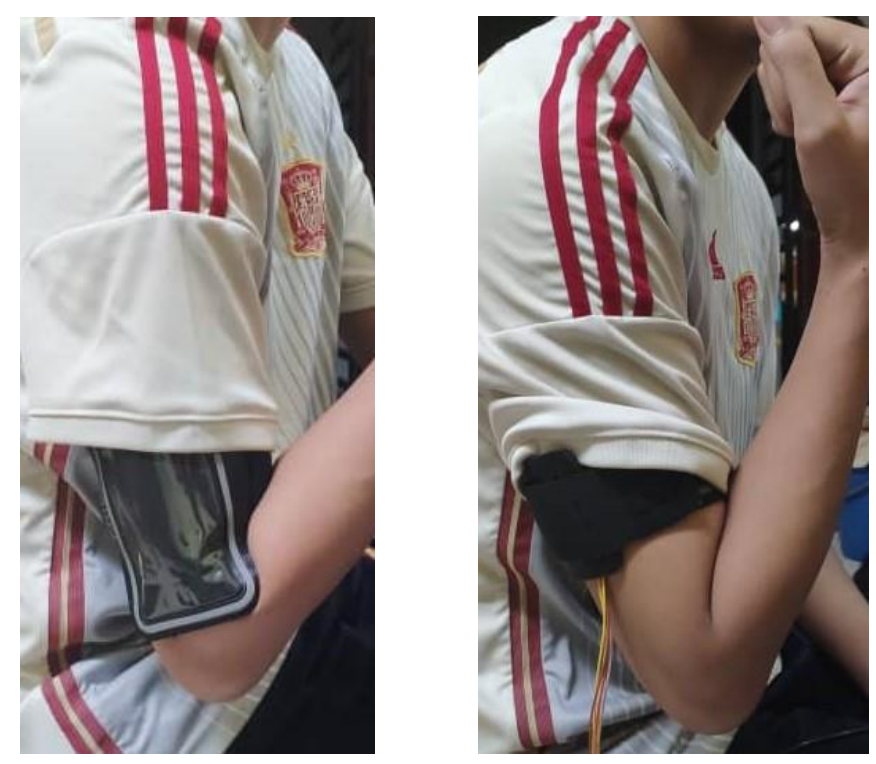

Figure 6. (a) Flexion for Biceps muscle (b) Flexion for Triceps muscle

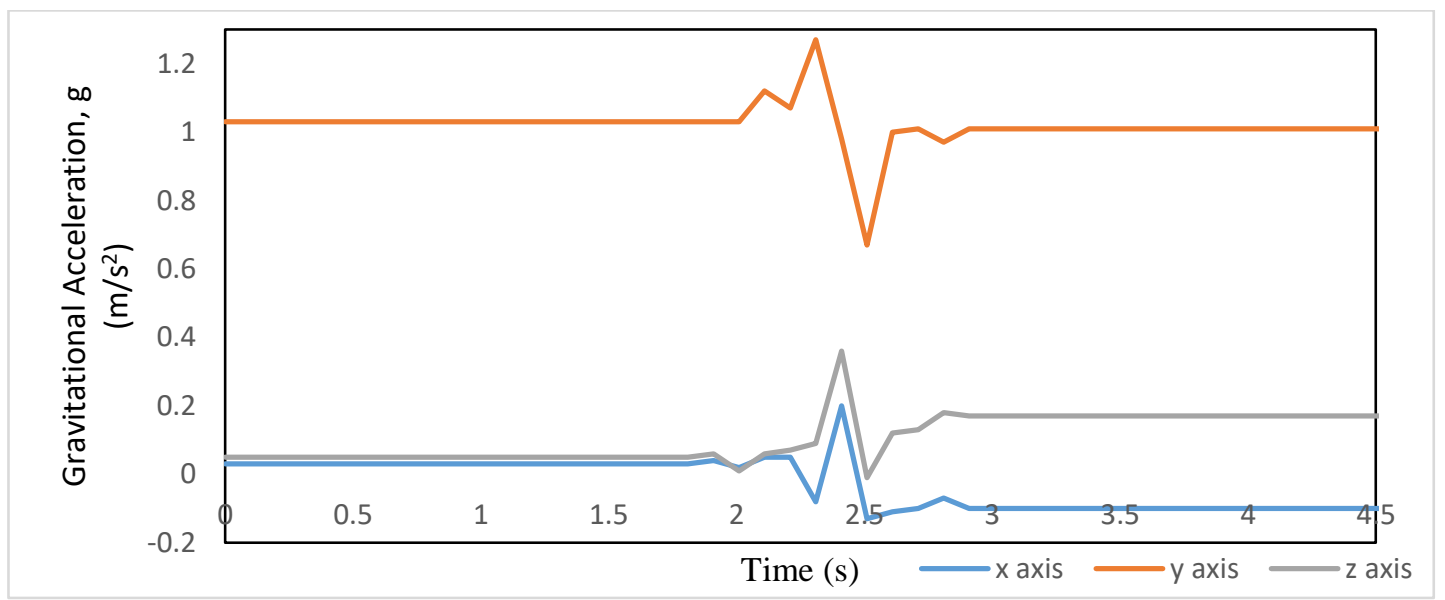

Figure 7. Signal for Flexion Biceps muscle

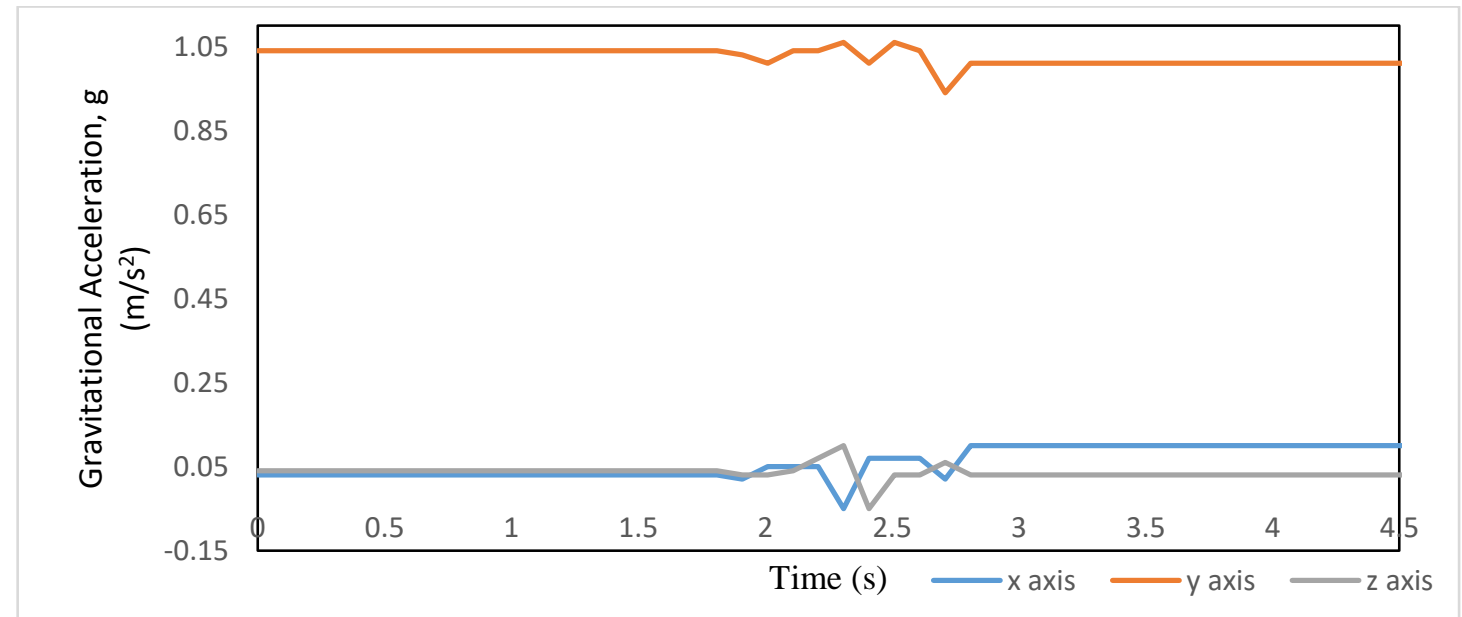

Figure 8. Signal for Flexion Triceps muscle

\section{Classification and Confusion Matrix}

There are two main parts for classification results which uses all features, and the other only for selected features in rank using Orange software based on Information Gain (IG) method. The features selected based on the IG method are MAV x-axis T, STD y-axis B, VAR y-axis B, RMS x-axis T and MAV x-axis B. The selection is made based on the best five. In Table $2, k$-NN shows $85.7 \%$ and $88.6 \%$ CA results for all selected features. 
Table 2. 10 fold CV on Training Set

\begin{tabular}{ccc}
\hline \multirow{2}{*}{ Model } & \multicolumn{2}{c}{ Classification Accuracy (CA) } \\
\cline { 2 - 3 } & All Features & Selected Features \\
\hline -NN & 0.857 & 0.886 \\
\hline
\end{tabular}

For training on the testing set in Table 3, the CA results for $k$-NN is $85.7 \%$ on all features while $88.6 \%$ on selected features.

Table 3. Training on Testing Set

\begin{tabular}{ccc}
\hline \multirow{2}{*}{ Model } & \multicolumn{2}{c}{ Classification Accuracy $($ CA) } \\
\cline { 2 - 3 } & All Features & Selected Features \\
\hline $\boldsymbol{n y y}$ & 0.857 & 0.886 \\
\hline
\end{tabular}

\section{CONCLUSION}

This study presents a classification technique for elbow movements which are extension and flexion. The primary objective is to determine which classifiers are best to classify the elbow movements. From this study, the $k$-NN classifier is used to validate all the features. Features extracted from muscle signals: MAV, RMS, VAR and STD from the MMG device that considers all three axes (x,y and $\mathrm{z}$ ). It could be seen from the study that when the selected features obtained from the IG feature selection method were used for testing on testing data, the $k$-NN yielded the CA up to $88.6 \%$ in selected features. Moreover, if all features were used, the $k$-NN achieved $85.7 \%$ of CA. In conclusion, selected features classifier models give more CA than all features classifier results.

\section{ACKNOWLEDGEMENT}

\section{REFERENCES}

[1] F. Bill and M. G. Foundation, "Global, regional , and national burden of stroke, 1990 - 2016 : a systematic analysis for the Global Burden of Disease Study 2016," pp. 439-458, 2016.

[2] R. Soltani-zarrin, A. Zeiaee, R. Langari, and R. Tafreshi, "Challenges and Opportunities in Exoskeleton-based Rehabilitation," pp. 2-5.

[3] Y. Geng, L. Chen, L. Tian, G. Li, and S. Member, "Comparison of Electromyography and Mechanomyogram in Control of Prosthetic System in Multiple Lim b Positions," Proc. 2012 IEEE-EMBS Int. Conf. Biomed. Heal. Informatics, vol. 25, no. C, pp. 788-791, 2012.

[4] M. R. M. Irfan, N. Sudharsan, S. Santhanakrishnan, and B. Geethanjali, "A Comparative Study of EMG and MMG Signals for Practical Applications," vol. 21, 2011.

[5] W. K. Cheng, I. M. Khairuddin, A. P. P. A. Majeed, and M. A. M. Razman, "The Classification of Heart Murmurs: The Identification of Significant Time Domain Features," MEKATRONIKA, vol. 2, no. 2, pp. 36-43, Dec. 2020.

[6] I. M. Khairuddin, S. N. I. Sidek, A. P. P. A. Majeed, and A. A. Puzi, "Classifying Motion Intention from EMG signal: A kNN Approach," 2019 7th Int. Conf. Mechatronics Eng. ICOM 2019, Oct. 2019.

[7] M. Sarillee et al., "Classification of muscle fatigue condition using multi-sensors," Proc. - 5th IEEE Int. Conf. Control Syst. Comput. Eng. ICCSCE 2015, pp. 200-205, May 2016. 